Instituto Internacional de Investigación y Desarrollo Tecnológico Educativo INDTEC, C.A.

DOI: https://doi.org/10.29394/Scientific.issn.2542-2987.2019.4.E.4.68-83

OAI-PMH: http://www.indteca.com/ojs/index.php/Revista Scientific/oai

Artículo Original / Original Article

\title{
La asertividad como estilo de comunicación en la formación del sujeto educador
}

Autores: Jennifer Zurina Quiñonez Fuentes Universidad Miguel de Cervantes, UMC jennifer.zurina@,hotmail.com Talca, Chile http://orcid.org/0000-0003-3724-0182

Genaro Moyano Arcos Universidad Miguel de Cervantes, UMC genaro31@gmail.com

Talca, Chile

http://orcid.org/0000-0001-6526-5411

\section{Resumen}

Las instituciones de educación superior ocupan un lugar central en el proceso educativo de los individuos, puesto que en ellas se inicia y desarrolla la formación profesional del hombre. El presente artículo tuvo como sujetos de estudio a los docentes del proyecto de educación integral de la universidad nacional Experimental Rafael María Baralt la cual cuenta con un universo de 88 docentes que atienden a una población estudiantil de dos mil siete (2007) estudiantes. El objetivo de la investigación fue promover la asertividad como estilo de comunicación en la administración de las clases en el aula bajo el paradigma crítico reflexivo y una investigación de tipo acción participativa donde se interactúa con los sujetos de la muestra. Como conclusiones finales que la asertividad es una herramienta eficaz como modelo de comunicación que promueve el aprendizaje colaborativo, dándole integralidad a la formación de los individuos en su proceso aprendizaje permitiendo a los estudiantes desenvolverse en un estado de confianza y seguridad que se traduce en madurez emocional con el apoyo del sujeto educador.

Palabras clave: comunicación; formación; docente.

Cómo citar este artículo:

Quiñonez, J., \& Moyano, G. (2019). La asertividad como estilo de comunicación en la formación del sujeto educador. Revista Scientific, 4(Ed. Esp.), 68-83, e-ISSN: 2542-2987. Recuperado de: https://doi.org/10.29394/Scientific.issn.2542-2987.2019.4.E.4.68-83

Fecha de Recepción:

04-05-2019
Fecha de Aceptación:

11-07-2019
Fecha de Publicación: 05-09-2019 


\title{
Assertiveness as a communication style in the training of the educator subject
}

\begin{abstract}
Higher education institutions occupy a central place in the educational process of individuals, since they begin and develop the professional training of man. The present article had as subjects of study the teachers of the integral education project of the Rafael María Baralt National Experimental University which has a universe of 88 teachers that serve a student population of two thousand seven (2007) students. The objective of the research was to promote assertiveness as a communication style in the administration of the classes in the classroom under the reflexive critical paradigm and a participatory action type investigation where it interacts with the subjects of the sample. As final conclusions, assertiveness is an effective tool as a communication model that promotes collaborative learning, giving integrality to the training of individuals in their learning process, allowing students to develop in a state of trust and security that translates into emotional maturity. with the support of the educator subject.
\end{abstract}

Keywords: communication; training; teacher.

\section{How to cite this article:}

Quiñonez, J., \& Moyano, G. (2019). Assertiveness as a communication style in the training of the educator subject. Revista Scientific, 4(Ed. Esp.), 68-83, e-ISSN: 2542-2987. Recovered from: https://doi.org/10.29394/Scientific.issn.2542-2987.2019.4.E.4.68-83

Date Received:

04-05-2019
Date Acceptance:

$11-07-2019$
Date Publication: 05-09-2019 


\section{Introducción}

La educación universitaria está determinada por las capacidades y posibilidades que posea cada estudiante, dado que la formación se ve influenciada por los constantes cambios propis de cada organización, por lo cual se interrelacionan una serie de factores que van desde lo organizativo hasta lo personal y que dotan a cada individuo de identidad propia.

Partiendo de esto es necesario reflexionar sobre la importancia de la asertividad como estilo de comunicación en la educación universitaria, viéndola fundamentalmente desde el punto de vista de la interacción y de libertad y seguridad que ésta le brinda al estudiantado, pues esto prepara el terreno para entender, comprender, diferenciar y relacionar lo asertivo de lo efectivo así como también a reconocer cada uno de los factores que intervienen en la comunicación entre ellos; la percepción, los valores y propósitos, además de la libertad y seguridad para expresar ideas con responsabilidad y sentido crítico.

Tanto en la vida profesional como en la social y familiar, los hombres tienen necesidades permanentes de relacionarse con otras personas, relaciones estas, que muchas veces se tornan difíciles y hasta tortuosas, cuando se siente que es vulnerada la voluntad del individuo y que debe plegarse a otra que no comparte, esto produce frustración, insatisfacción y peor aún disminución de la autoestima e inseguridad para enfrentar situaciones adversas y solucionar problemas.

La presente investigación se llevó a cabo con los docentes y alumnos del Proyecto Educación Integral de la Universidad Nacional Experimental "Rafael María Baralt", (UNERMB), en la Costa Oriental del Lago, sede Ciudad Ojeda, Venezuela, ubicada en el municipio Lagunillas, avenida 41, parroquia Alonso de Ojeda, cuyo universo lo conforman ochenta y ocho (88) docentes para atender una población de dos mil siete (2007) estudiantes.

Es importante destacar la Universidad Experimental "Rafael María 
Baralt", es una universidad pública de carácter experimental que nació en Cabimas, el 15 de marzo del año 1982, en el transcurrir de 30 años se consolidó como la universidad de la costa oriental del lago estableciendo sedes en seis de los siete municipios que conforman la subregión petrolera del estado Zulia.

Esta casa de estudio tiene como misión impulsar el desarrollo el de esta pujante zona petrolera, a través de la capacitación y formación del continuo humano, preparando profesionales para la industria y para el crecimiento social, cultural y económico. Igualmente, su visión es generar cambios en los valores en los individuos tanto en lo individual como en lo social que les permita tener un sistema educativo de calidad con fácil acceso para todos de manera integral

El objetivo de la investigación fue promover la asertividad como estilo de comunicación en la administración de las clases en el aula de tal manera que se pueda contribuir para que las equivocaciones o miedos de los estudiantes al expresarse públicamente puedan autorregularse a través de técnicas comunicacionales, para lo cual se tomó como fundamento un contexto teórico y la metodología de acción participativa que permitió llegar a las reflexiones finales que se mencionan al final del artículo.

\section{Desarrollo}

Como contexto inicial se puede decir que la educación superior en Venezuela al igual que Latinoamérica, no escapa de la imperante necesidad de caminar al ritmo que los avances tecnológicos y las exigencias de un mundo cambiante y complejo exigen, al tiempo que ésta debe marchar en pro del desarrollo las naciones comprometiéndose a formar profesionales competitivos para el campo laboral pero no deshumanizados, la educación del siglo XXI debe promover el convivir en armonía con el entorno y con el propio yo, desear esa convivencia implica convertirse en ciudadanos del mundo, sin 
perder sus raíces culturales ni la identidad como nación.

En atención a lo que se ha planteado anteriormente, como una situación que requiere atención, se tomó como actores de la investigación a estudiantes del programa educación, proyecto profesionalización docente de la universidad nacional experimental Rafael María Baralt para mostrarles la asertividad como estilo de comunicación y como ésta le brinda al ser humano la oportunidad de expresar sentimientos, emociones y puntos de vista de manera congruente, clara y equilibrada.

Para Gago (2017): la asertividad, además, está ligada a la conducta, por lo que el entrenamiento asertivo no solo permite desarrollar actividades inherentes y propias del ser humano sino que le ayuda a mejorar sus relaciones con su entorno y a desarrollar habilidades sociales (pág. 16).

Otro aspecto importante es, que a través de la comunicación asertiva se forman hombres y mujeres con pensamientos críticos, tolerantes y sensibles a la experiencia de los demás, capaces de encontrar áreas de acuerdo, así como defender sus puntos de vista inclusive ante un facilitador sin irrespetarlo, en este sentido, educar es ayudar a madurar, a hacer personas críticas proporcionándole criterios para elegir con autonomía y libertad un proyecto de vida.

En este sentido, la comunicación y la asertividad para Serrano (2004): son habilidades sociales que permiten mejorar las relaciones interpersonales, para favorecer el trabajo en equipo y que les facilite el trabajo en equipo (pág. 12). En esta dirección, Zabalza (2004): plantea que el éxito que experimenta el estudiante en este proceso depende en gran parte de la manera como se relaciona con las demás personas y especialmente con los compañeros y que la base de estas relaciones es la comunicación. Sin embargo, muchas veces estas no son efectivas, sino que se refiere a las personas en términos pocos constructivos (pág. 30).

Planteado lo anterior, vale la pena considerar que al tratar con los 
demás se debe tener en cuenta, como toman la conversación, su autoestima, su nivel de aceptación en cuanto a la manera de ser tratados.

Desde esta perspectiva es necesario estar claro que la persona interactúa y participa activamente cuando trabaja motivado por sus necesidades intrínsecas; es decir, por su propia iniciativa. Esta experiencia permite asimilar y acomodar los nuevos aprendizajes, adaptándolos y aceptándolos para cambiar y modificar esquemas personales. En resumen, se puede visualizar la tabla 1 , donde se elaboró una serie de ejemplos para entender mejor los aspectos relacionados con la asertividad.

Tabla 1. Comunicación Asertiva.

\begin{tabular}{|c|c|c|}
\hline ASERTIVA & AGRESIVA & PASIVA \\
\hline $\begin{array}{l}\text { "Tú tienes derecho, yo tengo } \\
\text { derecho" }\end{array}$ & "Yo tengo derecho, tú..." & "Tú tienes..." \\
\hline $\begin{array}{l}\text { Directa, firme. } \\
\text { "¿Cómo crees que } \\
\text { podríamos..." }\end{array}$ & $\begin{array}{l}\text { Impone, interrumpe. } \\
\text { "Deberías hacer..." }\end{array}$ & $\begin{array}{l}\text { Vacilante, cortada. } \\
\text { "No te molestes..." }\end{array}$ \\
\hline $\begin{array}{l}\text { Mirada directa y franca. } \\
\text { Gesto firme. } \\
\text { Postura relajada. } \\
\text { Voz sin vacilación, adecuado. }\end{array}$ & $\begin{array}{l}\text { Mirada fija. } \\
\text { Gestos amenazantes. } \\
\text { Postura hacia adelante. } \\
\text { Volumen de voz elevado. }\end{array}$ & $\begin{array}{l}\text { Rehúye la mirada. } \\
\text { Movimientos nerviosos. } \\
\text { Postura recogida. } \\
\text { Volumen bajo. }\end{array}$ \\
\hline $\begin{array}{l}\text { Relaciones positivas. } \\
\text { Resuelve problemas. }\end{array}$ & $\begin{array}{l}\text { Viola derechos. } \\
\text { Crea tensión. }\end{array}$ & $\begin{array}{l}\text { No consigue los objetivos. } \\
\text { Pierde oportunidades. } \\
\text { Conflictos personales. }\end{array}$ \\
\hline
\end{tabular}

Fuente: Los Autores (2019).

\section{Metodología}

El paradigma de investigación es crítico reflexivo, con una metodología de investigación de acción participativa. Para Rosario (2012): el paradigma crítico reflexivo permite una investigación centrada en la reflexión de las causas y consecuencias de las acciones en la clase y trata de dar una herramienta que les permita tanto a alumnos como docentes a valorar su propia práctica. Lo anterior mediante el uso de la acción participativa que hace a los investigadores parte del proceso y desarrollo de la investigación pues 
está en contacto directo con los participantes de la misma (pág. 38).

\section{Conclusiones}

Para el desarrollo de las conclusiones se hará referencia a algunas teorías del aprendizaje como las siguientes:

4.1. Cognitivista: dentro de la teoría cognitivista, se encuentra la teoría de aprendizaje social o vicaria de Albert Bandura, caracterizado por la observación, imitación, o modelado, indica que una de las formas de aprender es mediante la observación de las conductas de las personas gracias a la capacidad que posee el individuo para representar en su mente lo que percibe con sus ojos.

4.2. Teoría socio histórica de Lev Vygotski: esta teoría introduce el concepto de zona de desarrollo próximo que no es más que la distancia que existe entre la zona de desarrollo real, lo que un individuo es capaz de hacer por sí mismos, y la zona de desarrollo potencial que guarda relación con lo que el sujeto es capaz de alcanzar con la ayuda de un adulto o de un congéneres con habilidades cognitivas superiores, este aprendizaje está íntimamente relacionado con las experiencias y conocimientos previos. Plantea que el conocimiento no es un objeto que se pasa de uno a otro, es algo que se construye por medio de la interacción social en la que el ser humano está inmerso.

4.3. Teoría del aprendizaje significativo de Ausubel: está definida como aquella donde el aprendizaje es significativo y eficaz mediante la incorporación de nuevos conocimientos que se incorporan de forma sustantiva en la estructura cognitiva de los individuos que le permite relacionar los conocimientos adquiridos con los nuevos.

4.4. Teoría de la comunicación: implica el manejo de un lenguaje eficiente, positivamente afirmativo donde la persona comunica efectivamente sin una relación de subordinación lo que siente y piensa, haciendo valorar y respetar 
sus ideas como persona, lo cual fortalece la familiaridad en el trato con las demás personas. Con ella se crea un lazo no sólo entre los individuos grupos o equipos, sino también entre las relaciones que se establecen entre los miembros, fortaleciendo además la motivación que cada uno posee hacia el logro de las metas que son altamente valoradas.

4.5. Teoría de la crítica social: esta teoría indica que en la educación existe una crisis de valores generada por los cambios sociales que ha experimentado el mundo entero, promoviendo un gusto desmedido por lo novedoso y por aquellos elementos que una persona o corriente promueve para generar uniformidad en los gustos de los individuos. En la teoría crítica social se presenta al hombre como una fuente de apoyo a la tecnología y sus novedades. Persiguiendo un falso bienestar donde se anula el pensamiento libre y personal, convirtiendo la comodidad en un bien deseado.

Para dar respuesta al propósito de la investigación que fue promover la asertividad como estilo de comunicación en la administración de las clases en el aula y basados en las teorías y conceptos mencionados, se propone una educación fundamentada en la libertad personal, la responsabilidad, y autorrealización. Tal es el caso de Nieto y Rodríguez (2010), quienes indican que:

Todas las posibilidades de realización del individuo, deben apuntar y atender la educación, deben estar sustentadas expresamente por la función crítica. Los autores del artículo coincidimos que realmente existe una crisis de valores y se debe dar una vital importancia a la función del ser humano que posibilite un cambio consciente en la sociedad, un espíritu crítico sustentado por una verdadera búsqueda de conocimiento, así como de un sentido profundo de vida, ese es el camino hacia el verdadero diálogo y el verdadero progreso (pág. 25).

Otro argumento que ayuda a dar respuesta al objetivo es el que hace 
referencia a cómo se ha transcurrido de una sociedad primitiva, a una tradicional, y a otra moderna, para ello plantea dos dimensiones una técnica y otra social, en las cuales la comunicación es fundamental en el proceso de formación del educando y del sujeto educador.

Para Habermas (2002): "la Teoría Crítica quiere redimir la dimensión social de ese entramado positivista, por medio de una teoría reflexiva, para llevar al hombre hacia una emancipación, liberadora y más humana" (pág. 52).

Dentro de las conclusiones se mencionan las bases legales que respaldan de forma institucional los elementos mencionados, dando asidero teórico y legal a cada uno de los planteamientos del artículo de investigación.

Inicialmente se menciona en la Constitución de la República Bolivariana de Venezuela (1999a), en el Capítulo VI, de los derechos culturales y educativos, que:

La educación es un derecho humano y un deber social fundamental, es democrática, gratuita y obligatoria...La educación es un servicio público y está fundamentada en el respeto a todas las corrientes del pensamiento, con la finalidad de desarrollar el potencial creativo de cada ser humano y el pleno ejercicio de su personalidad en una sociedad democrática basada en la valoración ética del trabajo y en la participación activa, consciente y solidaria en los procesos de transformación social, consustanciados con los valores de la identidad nacional y con una visión latinoamericana y universal (art. 102).

De acuerdo al artículo 102 de la Constitución de la República Bolivariana de Venezuela (1999b), la educación debe estar fundamentada en todas las corrientes del pensamiento, lo que está respaldado en el siguiente artículo:

Toda persona tiene derecho a una educación integral, de calidad, permanente, en igualdad de condiciones y oportunidades, sin más limitaciones que las derivadas de sus aptitudes, vocación y aspiraciones. La educación es obligatoria en todos sus niveles, desde el maternal hasta el nivel medio 
diversificado. La impartida en las instituciones del Estado es gratuita hasta el pregrado universitario. A tal fin, el Estado realizará una inversión prioritaria, de conformidad con las recomendaciones de la Organización de las Naciones Unidas (art. 103).

De acuerdo al artículo 103, todos los individuos tienen derecho a una educación de calidad, esto es confirmado a continuación, en este artículo de la Constitución de la República Bolivariana de Venezuela (1999c):

La educación estará a cargo de personas de reconocida moralidad y de comprobada idoneidad académica. El Estado estimulará su actualización permanente y les garantizará la estabilidad en el ejercicio de la carrera docente, bien sea pública o privada, atendiendo a esta Constitución y a la ley, en un régimen de trabajo y nivel de vida acorde con su elevada misión. El ingreso, promoción y permanencia en el sistema educativo, partidista o de otra, serán establecidos por ley y responderá a criterios de evaluación de méritos, sin injerencia partidista o de otra naturaleza no académica (art. 104).

De acuerdo al artículo 104, los docentes deben estar calificados para impartir las clases, en este caso, se habla de la importancia de la profesionalización de la docencia.

Todo lo anterior, es respaldado por la Organización de las Naciones Unidas para la Educación, la Ciencia y la Cultura (UNESCO, 1998): donde se habla que la calidad de la educación y se hace referencia a que esta lleva consigo la mejora en las actividades académicas abarcando el proceso de enseñanza aprendizaje, la dotación y tecnologías y el desarrollo docente en todas sus esferas (pág. 1).

Para cumplir con lo expuesto, las instituciones educativas a nivel superior pretenden diseñar políticas de formación que permitan el desarrollo continuo y permanente de sus profesionales para mejorar las competencias, estimulando la innovación dentro del sistema pedagógico. Dicha necesidad obedece a un rol transformador de la educación a nivel superior en la sociedad 
a nivel laboral y académico en el contexto de la sociedad del conocimiento.

Como conclusión se puede decir que la asertividad es un estilo de comunicación claro que permite a estudiantes y docentes manifestar sus pensamientos manifestando la defensa de sus derechos sin perjudicar a otros individuos.

En este contexto, González (2011): respalda esta afirmación alegando que la asertividad permite defender convicciones sin someterse a voluntades ajenas, permite el desarrollo de habilidades sociales que le permiten la interacción con otras personas, lo cual es importante para fomentar el aprendizaje significativo en cada uno de los individuos involucrados dentro del proceso educativo (pág. 82).

Asimismo, Müller (2005): habla de los docentes tutores y los define como un individuo que coopera con otros para el desarrollo de habilidades y destrezas cognitivas, con el propósito que mejore, progrese y perfeccione su condición humana, para educar y auto-educarse (pág. 16); un sujeto educador inspira su entorno y es capaz de establecer relaciones sin dificultades y de construir nuevos conceptos, que le permitan participar de forma activa y en correspondencia con las exigencias de un mundo complejo y cambiante, manteniendo siempre despierta su conciencia sobre el fin y razón de ser de su acción educativa, fomentando el ser en sí que es la esencia de cada hombre.

Según documento de la Cámara Venezolana de Educación Privada CAVEP (2014), citado por la autora Arrieche (2018), menciona que:

Ofrecer una educación de calidad implica la articulación gobierno, educación, sociedad y familia, y se caracteriza por la transmisión de formas de actuar democrática, de fraternidad, basada en la pedagogía del aprendizaje significativo, donde se enseñe y se aprenda en y desde la libertad, que genere calidad de vida con visión de país a partir de un proyecto de Nación que es compartido por las mayorías (pág. 369).

Así, se puede decir que el resultado de una conducta asertiva no tiene 
siempre como derivación la ausencia del conflicto antagónico entre las partes, puede que esta cause molestias, pero cuando se actúa asertivamente al comunicar sentimientos, es satisfactorio poder expresar la opinión sin agredir a nadie, situación ésta que contribuye significativamente a disminuir la ansiedad a través de relaciones más íntimas y significativas, logrando mayor respeto a uno mismo y mejor adaptación social.

Luego de exponer las ideas se puede concluir que es posible promover la asertividad como medio de comunicación en el aula de clases y fuera de ella, tomando como punto de partida el respeto hacia los demás evitando desacuerdos en el entorno educativo y desarrollando individuos que, a partir de los conocimientos y experiencias previas, puedan participar responsablemente como seres que piensan, razonan, reflexionan y aportan ideas en la construcción de un aprendizaje significativo.

Finalmente, cuando se habla de asertividad como modelo de comunicación se promueve el aprendizaje colaborativo, lo que aporta mecanismos para aprovechar el conocimiento y darle integralidad a la formación de los individuos en su proceso aprendizaje, además se incluye el desarrollo de otros modelos mentales que les permiten a los estudiantes desenvolverse en un estado de confianza y seguridad que se traduce en madurez emocional.

\section{Referencias}

Arrieche, M. (2018). Gestión Docente en el Contexto de la Educación Primaria Venezolana. Revista Scientific, 3(7), 354-373, e-ISSN: 25422987. Recuperado de: https://doi.org/10.29394/Scientific.issn.2542$\underline{2987.2018 .3 .7 .18 .354-373}$

Constitución de la República Bolivariana de Venezuela (1999a,b,c). Capítulo VI, de los Derechos Culturales y educativos. Gaceta Oficial Extraordinaria $\mathrm{N}^{\circ} 36.860$ de fecha 30 de diciembre. Caracas, 
Venezuela: Asamblea Nacional Constituyente. Recuperado de:

https://www.oas.org/dil/esp/constitucion venezuela.pdf

Gago, M. (2017). Manual: Comunicación, asertividad y escucha activa en

la empresa. ISBN: 978-84-681-7712-0; ISBN: 978-84-681-8564-4.

Madrid, España: Editorial CEP, S.L. Recuperado de:

https://books.google.com.ec/books?id=LuxCDwAAQBAJ\&lpg=PP1\&hl

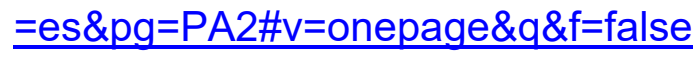

González, S. (2011). Habilidades de comunicación escrita: Asertividad.

Persuasión. Alto impacto. ISBN: 978-1-60255-376-7. Nashville,

Estados Unidos: Grupo Nelson, Inc - Thomas Nelson, Inc.

Habermas, J. (2002). Acción comunicativa y razón sin transcendencia.

ISBN: 9788449313080 . España: Paidós Ibérica.

Müller, M. (2005). Docentes tutores. Spanish Edition, Series: Educación y

Creatividad, ISBN 10: 950-507-511-1; ISBN 13: 978-9505075119.

Buenos Aires, Argentina: Editorial Bonum.

Nieto, S., \& Rodríguez, J. (2010). Investigación y evaluación educativa en

la sociedad del conocimiento. 1ra. Edición, ISBN: 978-84-7800-226-

9. España: Ediciones Universidad de Salamanca.

Rosario, V. (2012). Casos de Investigación e Innovación: Procesos para la Transformación de las prácticas e instituciones educativas. ISBN: 978-1-4633-1550-4; ISBN: 978-1-4633-1552-8; ISBN: 978-1-46331551-1. Estados Unidos: Red de Académicos de Iberoamérica; Instituto para el desarrollo de la Investigación e Innovación Educativa en Iberoamérica, S.C.

Serrano, S. (2004). El regalo de la comunicación. ISBN 10: 8433962027; ISBN 13: 9788433962027 . Barcelona, España: Editorial Anagrama.

UNESCO (1998). Declaración Mundial sobre la Educación Superior en el siglo XXI: Visión y Acción. Conferencia Mundial sobre la Educación Superior en el siglo XXI: Visión y acción, Código del documento: 


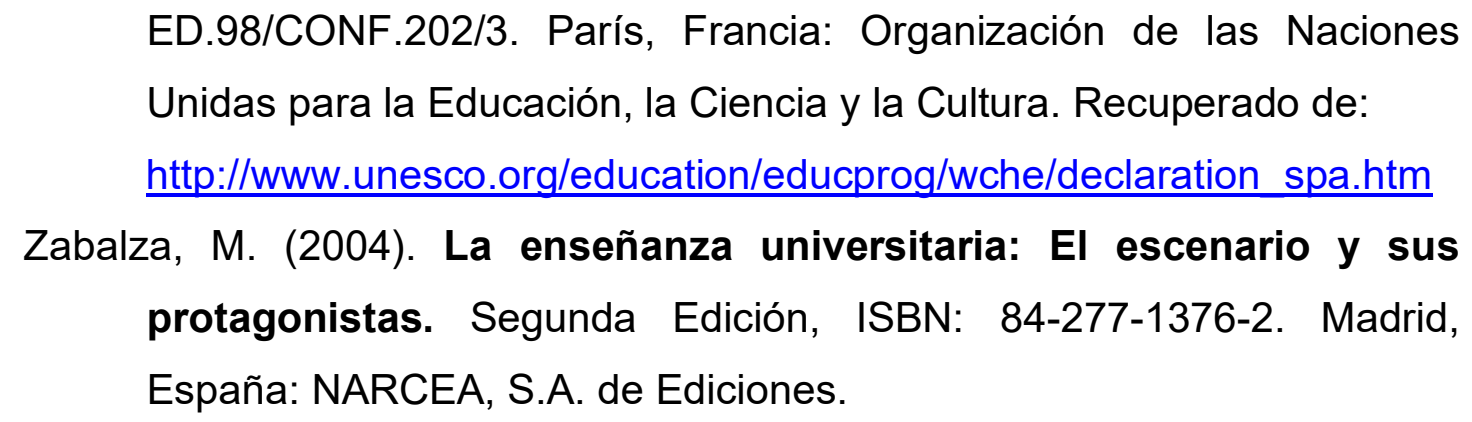

Unidas para la Educación, la Ciencia y la Cultura. Recuperado de:

http://www.unesco.org/education/educprog/wche/declaration spa.htm Esaña. NARCEA, S.A. de Ediciones. 


\section{Jennifer Zurina Quiñonez Fuentes}

e-mail: jennifer.zurina@hotmail.com 


\section{Genaro Moyano Arcos}

e-mail: genaro31@gmail.com

Nacido en Talca, Chile, el 08 de mayo del año 1976.

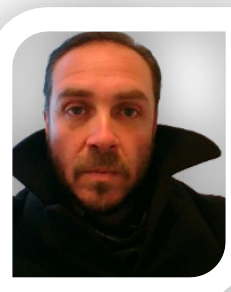

Magister en Lengua y Cultura; Magister en Gestión

Educativa; Licenciado en Comunicación Social;

Periodista; Académico en la Universidad Miguel de

Cervantes (UMC), Santiago, Chile; poseo además un

Postítulo en Beijing Yuyan Daxue, China, en Idioma y

Cultura; Docente en diversas entidades educativas del país; director académico y de Sede de instituciones de educación superior; Relator para instituciones gubernamentales; Centro de Desarrollo de Negocios, enfocados en el desarrollo de innovación y procesos de acompañamiento a pequeñas y medianas empresas; Encargado de talleres de formación en negociación para sindicatos.

El contenido de este manuscrito se difunde bajo una Licencia de Creative Commons ReconocimientoNoComercial-Compartirlgual 4.0 Internacional 